\title{
Recent Developments on Cylindrical Contact Force Models with Realistic Properties
}

\author{
F. Isaac ${ }^{1}$, F. Marques ${ }^{2}$, N. Dourado ${ }^{3}$ and P. Flores ${ }^{4}$ \\ ${ }^{1}$ CMEMS - University of Minho, Portugal, e-mail: efinhoisaac@hotmail.com \\ ${ }^{2}$ CMEMS - University of Minho, Portugal, e-mail: fmarques@dem.uminho.pt \\ ${ }^{3}$ CMEMS - University of Minho, Portugal, e-mail: nunodourado@dem.uminho.pt \\ ${ }^{4}$ CMEMS - University of Minho, Portugal, e-mail: pflores@dem.uminho.pt
}

\begin{abstract}
In this work a numerical study was performed involving a spatial revolute joint formed by a journal and a bearing with the purpose to evaluate the effect of the bearing thickness, misalignment and impact velocity on the force generated in the contact process. These analyses were run in a developed three-dimensional finite element model that simulates the referred aspects in a realistic way. This numerical tool allows defining both radial and axial clearances, which are important aspects in the analysis of operating conditions of actual journal-bearing joints. Resulting forces and stress profiles were calculated in different regions of the model, contributing to the accurate definition of design parameters. The generated data was ultimately compared and validated with the one obtained from the models that are currently used in the context of multibody dynamics and predict kinematic aspects of mechanical systems involving cylindrical contact interaction.
\end{abstract}

Key words: Cylindrical Contact Force; Finite Element Modeling; Radial and Axial Clearances; Misalignment; Impact Velocity

\section{Introduction}

Spatial revolute joints are mechanical components used to allow for the power transmission in many machines and mechanisms (Fig. 1(a)). These devices are commonly exposed to static and dynamic loadings that can be applied both to centered and misaligned elements. The analysis of the behavior of revolute joints is an important topic of research since it allows understanding the effect of several design features that may lead to a substantial reduction of the energy consumption and increase performance of many mechanical equipment.

Previous studies involving the numerical modeling of clearance joints (Fig. 1(b)) were performed considering a two-dimensional approach [1]. The results issued from those analyses are only valid for particular conditions that may not occur in most operating conditions. Indeed, when the third dimension is taken into account further aspects may be analyzed, such as the axial misalignment between the journal and the bearing, the effect of the axial (and radial) clearance, as well as the influence of asymmetric loading. In addition, a 3D-based numerical tool also allows analyzing 
the effect of the bearing thickness on the developed reaction force; the impact velocity of the journal on the bearing surface and how the members interact in a dynamic setting; as well as the friction phenomena associated with the contact surfaces. All of the cited aspects are at the origin of important operating non-conformities detected in many mechanical systems, such as vibrations, wear of functional surfaces and miss counterbalancing of the rolling members.

Once recognized that the realistic and accurate way to simulate clearance joints implies the consideration of the mentioned parameters and scenarios, it becomes crucial to choose an appropriate procedure to model such joints. This is possible to accomplish by means of finite element analysis (FEA), as it permits replicating accurately the interaction between the journal and the bearing. The post-processing of such analysis provides the developed contact force, as well as accurate cartographies of stresses, strains and displacements in any region of the model.

In the present work a numerical tool was developed to analyze the operating conditions of a common spatial revolute joint with radial and axial clearances that may be found in many mechanical systems (Fig.1(a)). The results obtained from the numerical computations were compared with those from the models proposed by other authors (Johnson [2]; Radzimovsky [3]; Goldsmith [4]; Lankarani and Nikravesh [5]; Ciavarella and Decuzzi [6, 7]; Liu et al. [8] and C. Pereira et al. [1]) for the particular condition of quasi-static loading and perfect alignment.

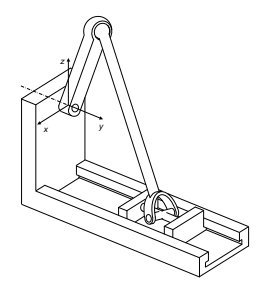

(a)

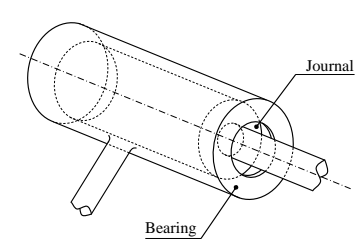

(b)

Fig. 1 Representation of a mechanism (a) and a spatial revolute joint (b)

\section{Finite Element Model for Spatial Revolute Joints}

In this study 8-node brick and 6-node prims (vicinity of the journal axis) elements were chosen to perform the FEA of the functional interaction between the journal and the bearing (Fig. 2). The model was constructed to facilitate the modification of geometrical parameters that characterize the model, providing the user a wide variety of design scenarios. The bearing and journal were both simulated as linear elastic materials ( $E=207 \mathrm{GPa}$ and $v=0.3$ ), with $\rho=7800 \mathrm{~kg} \cdot \mathrm{m}^{-3}$.

In the first loading increment both geometrical models were put in contact with each other. Most of the simulations were performed under quasi-static loading con- 
ditions, except an single study involving the manipulation of the velocity of the journal that was forced to move towards the bearing surface after being launched from the joint center. The boundary conditions were selected to simulate the operating conditions of spatial revolute joints in a realistic way. Hence, the nodes composing the external upper half of the bearing were fixed, while the set of nodes defining the journal axis were forced to move along the radial direction, of extent set equal to $0.05 \mathrm{~mm}$. During this process, the overall contact force was continuously monitored in the nodes that form the axis of the journal. Contact conditions were established at the interfaces of each body to prevent interpenetration, using an interfacial stiffness parameter characteristic of the used material joint.

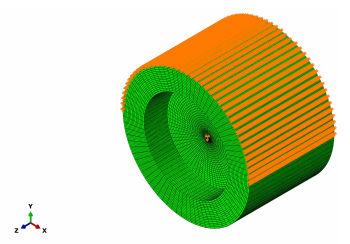

(a)

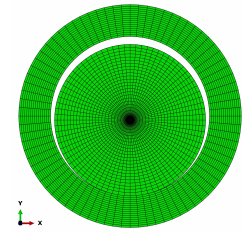

(b)

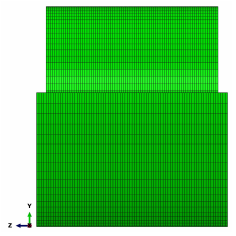

(c)

Fig. 2 FE mesh of the analyzed spatial revolute joint (a) with radial (b) and axial (c) clearances

A mesh convergence study was conducted to determine the required refinement level that permits attaining the necessary accuracy of the computed contact force between the journal and the bearing. In this regard, it was verified that the global force is predominantly affected by the discretization in the radial direction. Concerning the geometrical parameters, the radius of the bearing $\left(R_{\mathrm{B}}\right)$ and the length $\left(L_{\mathrm{B}}\right)$ were set equal to $10.0 \mathrm{~mm}$ and $20.0 \mathrm{~mm}$, respectively. Also, radial and axial clearances $\left(c_{\mathrm{r}}\right.$ and $\left.c_{\mathrm{a}}\right)$ were considered equal to $0.5 \mathrm{~mm}$ and $5.0 \mathrm{~mm}$, respectively. As $c_{\mathrm{r}}=\left(R_{\mathrm{B}}\right.$ $\left.R_{\mathrm{J}}\right)$ and $c_{\mathrm{a}}=\left(L_{\mathrm{B}} / 2-L_{\mathrm{J}} / 2\right)$, then the journal radius and length turns $R_{\mathrm{J}}=9.5 \mathrm{~mm}$ and $L_{\mathrm{J}}=10.0 \mathrm{~mm}$. The bearing thickness $t$ was set equal to $5 \mathrm{~mm}$.

Figure 3 shows the variation of the contact force (in percentage) with the size of the finite element used to discretize the bearing along the radial direction (from 2.5 $\mathrm{mm}$ to $0.15625 \mathrm{~mm}$, dividing the first one by a factor of 2), for different increments of the prescribed displacement. The criterion utilized to select the most adequate mesh size resulted from a trade-off between the overall computation time and the level of accuracy for the present analysis. Therefore, the model that accomplished this compromise in a better way corresponds to the one that present an element size of $0.3125 \mathrm{~mm}$ in the bearing region. This model is constituted by $281,6003 \mathrm{D}$ solid elements with a total of $1,5649,613$ nodes. These values enlighten the computational effort that the third dimension have introduced (24 hours on average).

Once attained the necessary mesh refinement, a study was conducted to analyze the effect of the bearing thickness, misalignment and initial velocity of the journal on the attained contact force. 


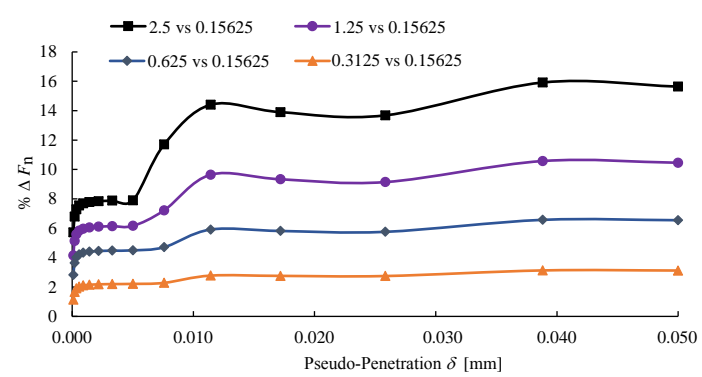

Fig. 3 Variation of the contact force for different element sizes

\section{Results and Discussion}

Numerical simulations were performed for the following conditions: $R_{\mathrm{B}}=10.0 \mathrm{~mm}$, $L_{\mathrm{B}}=20.0 \mathrm{~mm}, R_{\mathrm{J}}=9.5 \mathrm{~mm}, L_{\mathrm{J}}=18.0 \mathrm{~mm}, c_{\mathrm{r}}=0.5 \mathrm{~mm}$ and $c_{\mathrm{a}}=1.0 \mathrm{~mm}$.

Most of the models previously developed to study the contact interaction between journal and bearing assumed that the latter is merely a cylindrical surface of zero thickness. This hypothesis is not realistic because the components are solid and might be treated as such. Consequently, a study was made to analyze the influence of the bearing thickness $(t)$ on the contact forces $\left(F_{\mathrm{n}}\right)$, which permit to evaluate the contact stiffness $(K)$. Figure 4 shows the plotting of $F_{\mathrm{n}}$ for $t=2,4,6,8$ and 10 $\mathrm{mm}$. Each plot is presented with its corresponding regression of the type $F_{\mathrm{n}}=K \delta^{n}$ in order to identify the variation on the contact stiffness (coefficients of regression were higher than 0.999). The results are in agreement with what should be expected to occur, as they show the increase of the contact stiffness with the bearing thickness $t$ (except in the transition from $t=6 \mathrm{~mm}$ to $t=8 \mathrm{~mm}$, being that compensated with the decrease of the exponent $n$ from 1.03 to 1.01). This means that the required force to produce the same pseudo-penetration is larger in thicker bearings than in thinner ones. In further simulations, a bearing with $4 \mathrm{~mm}$ of thickness will be considered.

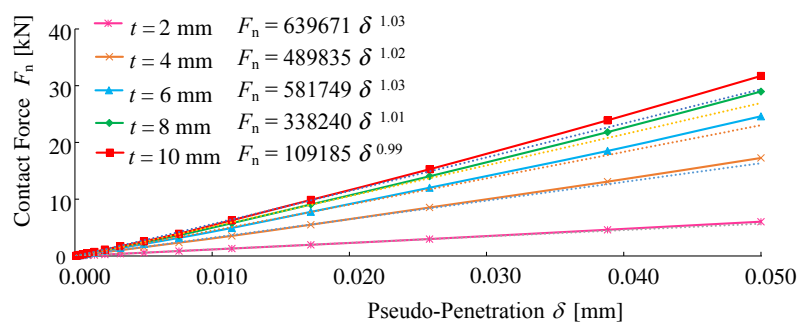

Fig. 4 Influence of the bearing thickness on the contact force $F_{\mathrm{n}}: c_{\mathrm{r}}=0.5 \mathrm{~mm}$ and $c_{\mathrm{a}}=1.0 \mathrm{~mm}$

Real mechanisms present imperfections in their components, which imply misalignment in their joints. The consequence associated with this phenomenon may 
lead to nonuniform wear of their members and consequent instabilities, which are at the origin of vibration and noise. In this work the misalignment of the cylindrical contact is defined in terms of the angle $\theta$, formed by the longitudinal axes (Fig. 5). Hence, a total of five amplitudes were considered: $\theta=1.0^{\circ}, 1.5^{\circ}, 2.0^{\circ}, 2.5^{\circ}$ and $\theta_{\max }$, with $\theta_{\max }$ written as follows,

$$
c_{\mathrm{r}}+R_{\mathrm{J}}\left(1-\cos \theta_{\max }\right)-\left(L_{\mathrm{J}} / 2\right) \sin \theta_{\max }=0
$$

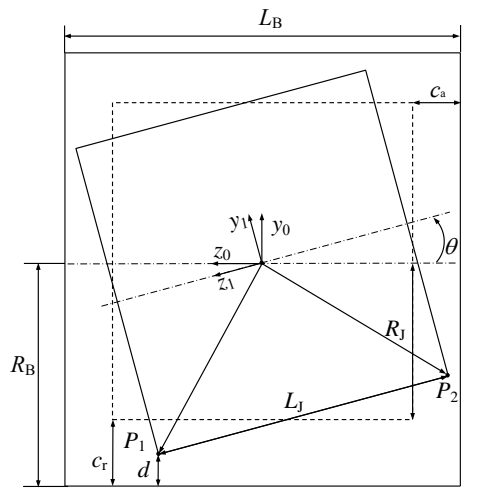

(a)

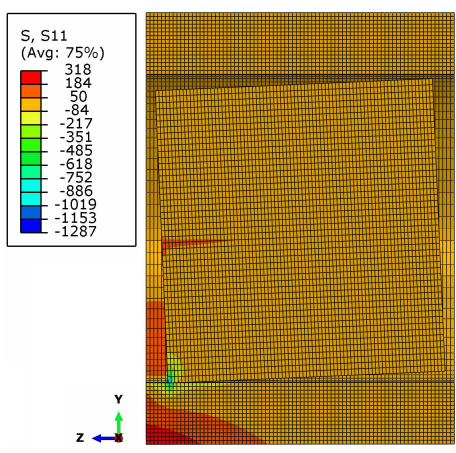

(b)

Fig. 5 Misalignment schematics (a) and FE misaligned configuration for $\theta=2.5^{\circ}$ (b)

For each value of $\theta$, a single node of the journal $\left(P_{1}\right)$ was firstly put in contact with the bearing surface (i.e., $d=0$ ), assuring that the journal is centered with the bearing. Then, a prescribed vertical displacement $(\delta=0.05 \mathrm{~mm})$ was applied to the nodes that compose the journal axis. The issued contact/reacting force on the interface of both bodies along with the corresponding potential regression are plotted in Fig. 6. It turns clear that for the same pseudo-penetration $\delta$ the increase of $\theta$ is followed by the decrease in the contact force and contact stiffness (it should be noticed that in the transition from $\theta=1.5^{\circ}$ to $2.0^{\circ}$ the exponent $n$ increases). These results are consistent with the thought that for higher misalignments the contact area between both bodies decreases that is, less material to react mechanically.

The effect of the initial velocity of the journal on the contact force generated by the contact with the bearing was investigated with the same premises as the ones established for the previous studies (i.e., $R_{\mathrm{B}}=10.0 \mathrm{~mm}, L_{\mathrm{B}}=20.0 \mathrm{~mm}, R_{\mathrm{J}}=9.5$ $\mathrm{mm}, L_{\mathrm{J}}=18.0 \mathrm{~mm}, c_{\mathrm{r}}=0.5 \mathrm{~mm}$ and $c_{\mathrm{a}}=1.0 \mathrm{~mm}$ ), considering the following initial velocities: $v=0.05,0.08,0.10,0.15$ and $0.20 \mathrm{~m} / \mathrm{s}$. In contrast with the previous simulations, the starting position of the journal model was coincident with the bearing axis, which enable mimicking the inertial effect of the journal $\left(\rho=7800 \mathrm{~kg} \cdot \mathrm{m}^{-3}\right)$.

Figure 7 allows observing the influence of the starting (release) velocity of the journal model on the reacting force generated in the contact with the bearing surface, for each monitored pseudo-penetration. As it was expected, the maximum contact 


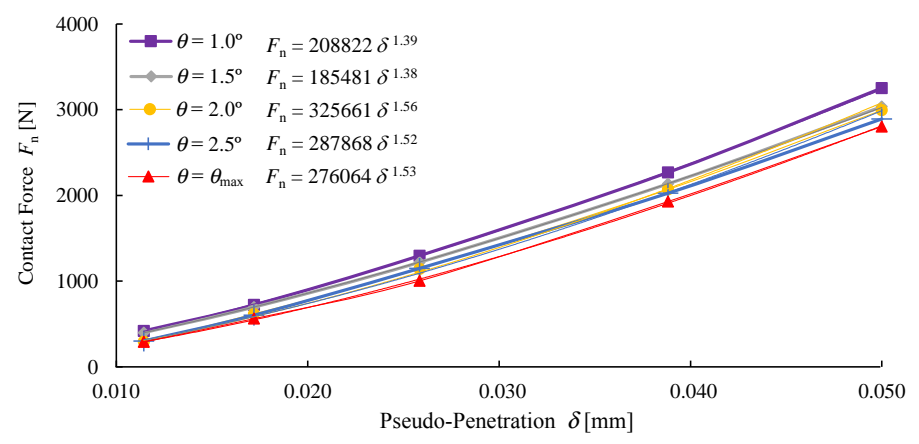

Fig. 6 Effect of the misalignement on the contact force $F_{\mathrm{n}}: c_{\mathrm{r}}=0.5 \mathrm{~mm}$ and $c_{\mathrm{a}}=1.0 \mathrm{~mm}$

force increases with the initial velocity, which is followed by an increase of the attained maximum displacement when the journal reaches the zero velocity. It is shown that the evolution of the contact force with the attained pseudo-penetration is clearly non-linear, which contrasts with the trend observed when the journal is subjected to quasi-static loading (Fig. 4). This discrepancy increases with the mass of the journal, due to the inertia of the journal member.

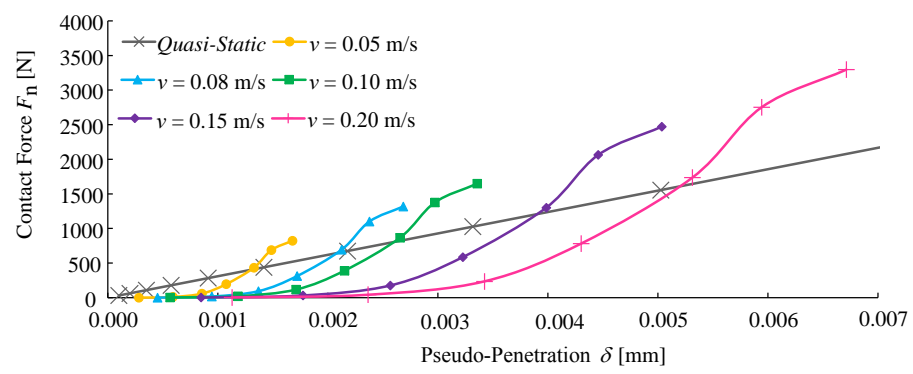

Fig. 7 Effect of the initial velocity of the journal on the contact force $F_{\mathrm{n}}$ and on the psudopenetration $\delta: c_{\mathrm{r}}=0.5 \mathrm{~mm}$ and $c_{\mathrm{a}}=1.0 \mathrm{~mm}$

The results issued from this study were compared with analytical approaches currently used in the Multibody Dynamics formulation, for the same conditions (i.e., $R_{\mathrm{B}}=10 . .0 \mathrm{~mm}, L_{\mathrm{B}}=20.0 \mathrm{~mm}, R_{\mathrm{J}}=9.5 \mathrm{~mm}, L_{\mathrm{J}}=18.0 \mathrm{~mm}, c_{\mathrm{r}}=0.5 \mathrm{~mm}$ and $c_{\mathrm{a}}$ $=1.0 \mathrm{~mm}$ ), with a maximum prescribed displacement $\delta=0.05 \mathrm{~mm}$ (Fig. 8). The axis of the journal and the bearing were aligned with each other, which corresponds to the configuration of $\theta=0^{\circ}$. In the analyses the compressive normal forces were also evaluated along the axis of the journal. The cylindrical models that were considered in this analysis are the ones proposed by Johnson, Radzimovsky, Goldsmith, Lankarani and Nikravesh (L\&N), Ciavarella and Decuzzi (Persson's theory), Liu et 
$a l$. and C. Pereira et al.. The results obtained in the present work are in agreement with the ones plotted with models that used FEA.

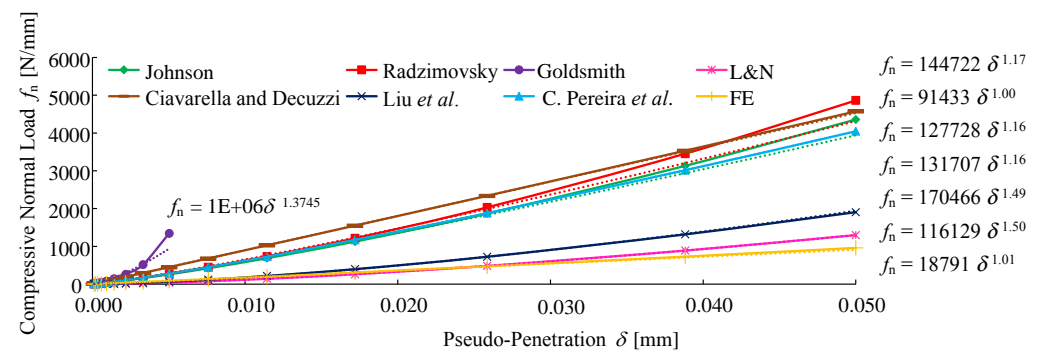

Fig. 8 Comparisson of the normal compression load $f_{\mathrm{n}}$ obtained from the finite element model developed and from the current analytical cylindrical contact models employed in Multibody Dynamics $(\mathrm{MBD}): c_{\mathrm{r}}=0.5 \mathrm{~mm}$ and $c_{\mathrm{a}}=1.0 \mathrm{~mm}$

Figure 9 shows the cartographies of the normal stresses obtained with aligned and misaligned models for the same value of the bearing thickness (i.e., $t=4 \mathrm{~mm}$ ) and $c_{\mathrm{r}}=0.5 \mathrm{~mm}$ and $c_{\mathrm{a}}=1 \mathrm{~mm}$. The stress fields are in agreement with the expectable scenario of a contact interaction between two solid bodies.

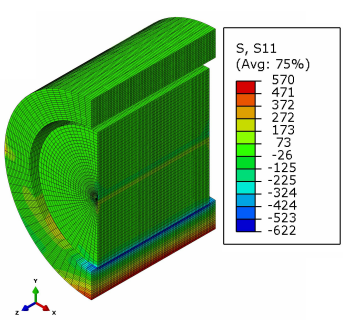

(a)

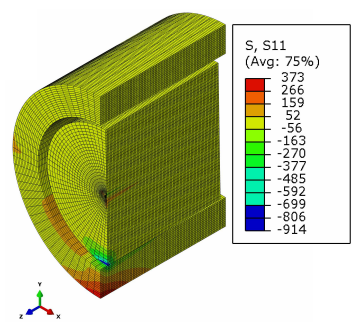

(b)

Fig. 9 Stress fields (normal stresses) installed in the spatial revolute joint for the aligned (a) and misaligned (b) scenarios

\section{Concluding Remarks}

An analysis of the mechanical behavior of a spatial revolute joint with both radial and axial clearances was performed to investigate the effect of the bearing thickness, misalignment and the velocity of the journal when this is moved towards the bearing surface. These joints are usually submitted to asymmetric loading or present geo- 
metrical misalignments in their axes, the more realistic manner to undergo numerical simulations is through a $3 \mathrm{D}$ model. Also, the possibility to evaluate the stress and strain fields of both bodies in their entire extent, and the ability to simulate the radial and axial clearances constitute aspects that are only possible to accomplish with success by means of a three-dimensional model.

The results obtained from computational simulations showed a coherent trend both for the static and dynamic loading scenarios. This has been corroborated both by the deformation of the interacting models and the trends of the contact force for different values of bearing thickness, angle of misalignment and initial velocities. It has been concluded that the increase of the bearing thickness has led to the increase of the contact force and contact stiffness, for each monitored displacement. In addition, the increase of the misalignment of both models originated the decrease of the contact force and contact stiffness, which was explained by the reduction of the contact area of the joint. The simulation of a dynamic scenario allowed concluding that the psudo-penetration in the journal-bearing interface increased with the impact velocity, which was triggered by the inertial effect of the journal. Finally, a comparison of the issued results has been made using the Multibody Dynamics formulation, revealing an agreement with those that used FE analyses. For future developments, the authors aim to study the impact of implementing this approach in the modeling of spatial revolute joints with clearance which can be attained through the analysis of the dynamic response of a multibody mechanical system.

Acknowledgements The second author expresses his deepest gratitude to the Portuguese Foundation for Science and Technology through the PhD grant (PD/BD/114154/2016). This work is supported by FCT with the reference project UID/EEA/04436/2013, by FEDER funds through the COMPETE 2020 Programa Operacional Competitividade e Internacionalização (POCI) with the reference project POCI-01-0145-FEDER-006941.

\section{References}

1. Pereira, C., Ramalho and A., Ambrosio, J.: An enhanced cylindrical contact force model. Multibody Syst Dyn, 35, 277-298 (2015)

2. Johnson, K.L.: One hundred years of Hertz contact. Proc. Inst. Mech. Eng., 196, 363-378 (1982)

3. Radzimovsky, E.I.: Stress distribution and strength conditions of two rolling cylinders pressed together. Eng. Exp. Sta. Univ. Ill. Bull., 408, (1953)

4. Goldsmith, W.: Impact, The Theory and Physical Behaviour of Colliding Solids. Edward Arnold, Sevenoaks (1960)

5. Lankarani, H.M. and Nikravesh, P.E.: A contact force model with hysteresis damping for impact analysis of multibody systems. Journal of Mechanical Design, 112(3), 369-376 (1990)

6. Ciavarella, M. and Decuzzi P.: The state of stress induced by the plane frictionless cylindrical contact 1: the case of elastic similarity. Int. J. Solids. Struct., 38, 4507-4523 (2001)

7. Ciavarella, M. and Decuzzi P.: The state of stress induced by the plane frictionless cylindrical contact 2: the general case (elastic dissimilarity). Int. J. Solids. Struct., 38, 4523-4533 (2001)

8. Liu, C.-S., Zhang, K. and Yang, R.: The FEM analysis and approximate model for cylindrical joints with clearances. Mech. Mach. Theory, 42, 183-197 (2007) 